\title{
ON THE SMITH NORMAL FORM OF THE VARCHENKO BILINEAR FORM OF A HYPERPLANE ARRANGEMENT
}

\author{
Graham Denham and Phil Hanlon
}

To the memory of Olga Taussky-Todd

\section{Terminology and Notation.}

Let $\mathcal{A}=\left\{H_{1}, \ldots, H_{\ell}\right\}$ be an arrangement of hyperplanes in $\mathbf{R}^{n}$ and let $r(\mathcal{A})=\left\{R_{1}, \ldots, R_{m}\right\}$ denote the set of regions in the complement of the union of $\mathcal{A}$. Let $L(\mathcal{A})$ denote the collection of intersections of hyperplanes in $\mathcal{A}$ including the empty intersection which we take to be $\mathbf{R}^{n}$. We order the elements of $L(\mathcal{A})$ by reverse inclusion thus making it into a poset. It is well known that this poset is a semilattice and is a geometric lattice if the arrangement is central. We will abbreviate $L(\mathcal{A})$ to $L$ when the arrangement is clear.

For regions $S, T \in R(\mathcal{A})$, define $n(S, T)$ to be the number of hyperplanes in $\mathcal{A}$ which separate $S$ from $T$. In [6], Varchenko defines a matrix $B=B(\mathcal{A})$ with rows and columns indexed by the regions in $R(\mathcal{A})$ by saying that the $S, T$ entry in $B$ is $q^{n(S, T)}$.

Example 1.1. An important example is the arrangement $\mathcal{A}$ consisting of the $\left(\begin{array}{l}n \\ 2\end{array}\right)$ hyperplanes $H_{i, j}$ in $\mathbf{R}^{n}$ given by

$$
H_{i, j}=\left\{\left(x_{1}, \ldots, x_{n}\right): x_{i}=x_{j}\right\} .
$$

The reader will note that $\mathcal{A}$ consists of the reflecting hyperplanes for the root system $\mathbf{A}_{n-1}$ and so we denote this arrangement by $\mathbf{A}_{n-1}$. Two points $\left(x_{1}, \ldots, x_{n}\right)$ and $\left(y_{1}, \ldots, y_{n}\right)$ are in the same region of the complement if and only if the relative orders of their coordinates are the same. So, the permutations in $S_{n}$ index the regions of the complement via the correspondence

$$
\sigma \leftrightarrow\left\{\left(x_{1}, \ldots, x_{n}\right): x_{\sigma 1}<x_{\sigma 2}<\ldots<x_{\sigma n}\right\} .
$$

For $\sigma, \tau$ in $S_{n}$, the exponent of $q$ in the $\sigma, \tau$ entry of $B$ is $i\left(\sigma \tau^{-1}\right)$, the number of inversions of $\sigma \tau^{-1}$. Equivalently, $B$ is the matrix for left multiplication by $\sum_{\alpha \in S_{n}} q^{i(\alpha)} \alpha$ in $\mathbf{C} S_{n}$ (the matrix with respect to the standard basis). It is interesting to note that this matrix is studied by Zagier [10] for quite different reasons. 
Example 1.2. For each $i$, let $O_{i}$ denote the linear hyperplane in $\mathbf{R}^{n}$ which consists of all vectors with a 0 in the $i^{t h}$ coordinate. Let $\mathbf{O}_{n}$ denote the arrangement $\left\{O_{1}, O_{2}, \ldots, O_{n}\right\}$. In this case, $r(\mathcal{A})$ has size $2^{n}$ and the individual regions can be indexed by sequences $S=\left(s_{1}, \ldots, s_{n}\right)$ where each $s_{i}$ is either +1 or -1 . The sequence $S$ corresponds to the region $R_{S}$ which contains all vectors $\left(x_{1}, \ldots, x_{n}\right)$ where $x_{i}<0$ if and only if $s_{i}=-1$. Given sequences $S, T$, the number of hyperplanes separating $R_{S}$ and $R_{T}$ is equal to the number of coordinates in which $S$ and $T$ differ.

Varchenko is interested in the bilinear forms corresponding to the matrices $B$. In papers [6] and [8], Varchenko and Schechtman show that these bilinear forms describe the analogue of Serre's relations for quantum Kac-Moody Lie algebras and are relevant to the representation theory of quantum groups and the study of the corresponding hypergeometric functions.

In these applications, it is the nullspace of $B$ that is of particular interest. Of course, this nullspace depends on the value of the parameter $q$. That motivates the following remarkable result of Varchenko which determines those values of $q$ for which $B$ is singular.

Theorem 1.3 (Varchenko [6, Theorem 1.1]). The determinant of the bilinear form of the configuration $\mathcal{A}$ is given by the formula

$$
\operatorname{det} B=\prod_{X \in L(\mathcal{A})^{*}}\left(1-q^{2 h(X)}\right)^{\ell(X)}
$$

where $L(\mathcal{A})^{*}$ is the set of non-empty intersections in $L(\mathcal{A})$, where $h(X)$ is the number of hyperplanes containing $X$ and where $\ell(X)$ is a non-negative integer.

In [6], Varchenko discusses two methods that can be used to compute the exponents $\ell(X)$. The first, which immediately precedes the statement of his Theorem 1.1, is geometric. The second which comes later in the paper (4.8) is more combinatorial. For completeness, we will briefly describe the second method. To compute $\ell(X)$, first choose a hyperplane $H \in \mathcal{A}$ which contains $X$. Then $2 \ell(X)$ is the number of regions $P$ which have the property that $X$ is the minimal edge $Y$ containing $\bar{P} \cap H$.

It is interesting to see what Theorem 1.3 says in the two special cases described above. First assume that $\mathcal{A}=\mathbf{A}_{n-1}$. This is a central arragement and so in this case the $L(\mathcal{A})$ is a lattice. It is well-known that $L(\mathcal{A})$ is the partition lattice $\Pi_{n}$. Given a partition $\alpha=\alpha_{1} / \alpha_{2} / \ldots / \alpha_{m}, \ell(\alpha)=0$ unless 
exactly one of the $\alpha_{i}$ has size greater than 1 . In that case, assume that the blocks are ordered so that $\left|\alpha_{1}\right|$ has size $a>1$ and that all other blocks have size 1 . Then $\ell(\alpha)=(a-2) !(n-a+1)$ !. This fact follows easily from Varchenko's description of $\ell$ discussed above. So in this case, we obtain the following factorization of $\operatorname{det}(B)$ which had originally been derived by Zagier [10]:

$$
\operatorname{det}(B)=\prod_{a=2}^{n}\left(1-q^{a(a-1)}\right)^{\left(\begin{array}{l}
n \\
a
\end{array}\right)(a-2) !(n-a+1) !} .
$$

Next assume that $\mathcal{A}=\mathbf{O}_{n}$. This is again a central arrangment and so $L(\mathcal{A})$ will again be a lattice. In this case, the lattice $L(\mathcal{A})$ is $B_{n}$, the lattice of subsets of $\{1, \ldots, n\}$. The indexing of edges by subsets is given as follows: the subset $U$ corresponds to the intersection of the $O_{u}$ for $u \in U$.

Using the method above for computing $\ell(X)$, it can be shown that

$$
\ell(U)= \begin{cases}2^{n-1}, & \text { if } U \text { is a hyperplane } \\ 0, & \text { otherwise }\end{cases}
$$

So in this case, $\operatorname{det}(B)=\left(1-q^{2}\right)^{n 2^{n-1}}$.

In this paper we will study the Smith Normal Form of the matrices $B$. Matrix theory is the subject that Olga wrote about more than any other. In fact, algebraic aspects of matrix theory were a particular interest of hers. So we feel that this paper is a fitting contribution to the special issue of the Pacific Journal of Math. in honor of Olga's work. The authors wish to thank Mel Hochster for many useful conversations during the course of this research.

\section{The Smith Normal Form of B.}

Both examples discussed in the previous section have the property that there is a group $G$ acting on the arrangement $\mathcal{A}$. Consider the situation where a finite group $G \subset G L_{n}\left(\mathbf{R}^{n}\right)$ acts on $\mathcal{A}$ hence on the vector space $V$ with basis consisting of $r(\mathcal{A})$. In this case, $G$ commutes with the matrix $B$ and so $B$ acts on each isotypic component of the action of $G$ on $V$ and $G$ acts on the kernel of $B$. Varchenko [6] and Zagier [10] independently ask what can be said about the determinant of $B$ on the isotypic components of $G$ on $V$ and what can be said about the action of $G$ on the kernel of $B$ for values of $q$ where $\operatorname{det}(B)$ vanishes.

In recent work, Hanlon and Stanley [2] consider these questions for the arrangement $\mathcal{A}=\mathbf{A}_{n-1}$ discussed in Example 1.1. In this work, they are led naturally to questions about the Smith Normal Form, over the ring $\mathbf{Q}[q]$, of 
the matrix $B$. These questions are the motivation for our investigation of the Smith Normal Form in this paper.

2.1. Computation of the Smith Normal Form. Let $\operatorname{SNF}(B)$ denote the Smith Normal Form of $B$ over $\mathbf{Q}[q]$. Recall that the Smith Normal Form is an invariant form for simultaneous left and right multiplication by unimodular matrices with entries in $\mathbf{Q}[q]$. It is known that $\operatorname{SNF}(B)$ is a diagonal matrix, $\operatorname{SNF}(B)=\operatorname{diag}\left\{d_{1}, \ldots, d_{n}\right\}$ where $d_{i} \mid d_{i+1}$ for each $i$. Our interest will be in the factors of the $d_{i}$ as polyomials in $q$. Let $\psi$ be an irreducible polynomial in $\mathbf{Q}[q]$. For each $i$, let $m_{i}(\psi)$ denote the multiplicity of $\psi$ as a factor of $d_{i}$, and let $M_{\psi}(x)$ be the generating function for the $m_{i}$, i.e., $M_{\psi}(x)=\sum_{i} m_{i}(\psi) x^{i}$. By Varchenko's Theorem, $M_{\psi}(x)=0$ unless $\psi$ is a cyclotomic polynomial.

Example 2.1. Let $\mathcal{A}=\left\{\ell_{1}, \ell_{2}\right\}$ be the arrangement in $\mathbf{R}^{2}$ given by the collection of the two parallel lines:

$$
\begin{aligned}
& \ell_{1}=\{(x, y): x=0\} \\
& \ell_{2}=\{(x, y): x=1\} .
\end{aligned}
$$

The complement $\mathbf{R}^{2}$ consists of three regions:

$$
\begin{aligned}
& R_{1}=\{(x, y): x<0\} \\
& R_{2}=\{(x, y): 0<x<1\} \\
& R_{3}=\{(x, y): x>1\}
\end{aligned}
$$

The matrix $B$ with respect to the regions as numbered above is:

$$
B=\left(\begin{array}{ccc}
1 & q & q^{2} \\
q & 1 & q \\
q^{2} & q & 1
\end{array}\right) .
$$

There is a standard algorithm for computing the Smith Normal Form for an $n \times n$ matrix $M$ over a Euclidean ring $A$ (see for example Newman [4]). The algorithm goes as follows. Let $\gamma$ denote the greatest common divisor of the elements in the first row of $M$. Find $a_{1}, \ldots, a_{n} \in A$ such that $\sum a_{i} m_{1, i}=$ $\gamma$. As proved in [4], it is possible to construct a unimodular matrix $E$ in $G L_{n}(A)$ with first column $\left[a_{1}, \ldots, a_{n}\right]^{t}$. Right multiplication of $M$ by $E$ leaves an equivalent matrix which has $\gamma$ in the 1,1 entry. Then right multiplication of this matrix by a suitable unimodular matrix will yield a matrix with first row $[\gamma, 0, \ldots, 0]$. At this point, use a similar method to produce via left unimodular multiplication, a matrix which has the greatest common divisor of the first column in its 1,1 entry and all other entries in 
column 1 equal to zero. This process may create non-zero entries in row 1 but will reduce (in the divisibility sense) the 1,1 entry. Repeat the process all over again until every entry of row 1 and column 1 is zero except the 1, 1 entry. Then compute the Smith Normal Form of the matrix in rows and columns $2-n$ by induction on $n$.

When we apply this algorithm to the matrix $B$ from Example 2.1 we go through the series of steps:

$$
\begin{aligned}
& \left(\begin{array}{ccc}
1 & 0 & 0 \\
q & 1-q^{2} & q-q^{3} \\
q^{2} & q-q^{3} & 1-q^{4}
\end{array}\right) \\
& \left(\begin{array}{ccc}
1 & 0 & 0 \\
0 & 1-q^{2} & q-q^{3} \\
0 & q-q^{3} & 1-q^{4}
\end{array}\right) \\
& \left(\begin{array}{ccc}
1 & 0 & 0 \\
0 & 1-q^{2} & 0 \\
0 & q-q^{3} & 1-q^{2}
\end{array}\right) \\
& \left(\begin{array}{ccc}
1 & 0 & 0 \\
0 & 1-q^{2} & 0 \\
0 & 0 & 1-q^{2}
\end{array}\right) \text {. }
\end{aligned}
$$

Note that some of the entries of the intermediate matrices have degree 4 which exceeds the maximum degree of any entry in either the original matrix or in the Smith Normal Form. This phenomena of degree "blow up" will be discussed at some length later in this section.

The conjectures put forth in this paper are based on the study of the Smith Normal Forms of the Varchenko $B$ matrices associated to a number of arrangements $\mathcal{A}$ in dimensions 2 to 5 . In theory, it should be straightforward to obtain this computational evidence using the standard algorithm discussed above. However, in practice the standard algorithm, directly applied, works very poorly. There are two problems. One problem is the size of $B$. The number of regions (hence the size of $B$ ) grows very quickly with the number of hyperplanes in the arrangement. For example, the $B$ matrix associated with the arrangement $\mathbf{A}_{5}$ is a 720 by 720 matrix with entries which are polynomials of degree 15. Computing the Smith Normal Form of a matrix of that size, using the standard algorithm, is infeasible. However, a second and more insidious problem is the blow-up of degrees of the matrix entries during intermediate stages in the computation. The matrices that one obtains in intermediate stages of the standard algorithm can have entries of very high degree. For example, one computation we performed consisted of finding the Smith Normal Form of a 9 by 9 matrix with entries which were polynomials 
of degree 15 or less. The symbolic mathematics package MAPLE ran for one week on a SPARC-20 without ever finishing this computation. At that point, we wrote our own code which would print out the matrices obtained at each step of the standard algorithm. After clearing the first row, the resulting matrix had certain entries in rows 2-9 which were of degree 30 . Then, after clearing the first column, some entries in rows 2-9 had degree close to 90 . Then after another first row clear, the degrees of some entries had exceeded 120. This growth of degrees at intermediate steps of the standard algorithm is our guess for what was slowing down the original MAPLE computation. This kind of problem is one that has been recognized in the computation of the Smith Normal Form of matrices over Z. George Havas [3] has done some excellent work on dealing with the analogous problem over the ring $\mathbf{Z}$.

We are going to discuss two methods we used to improve on the standard algorithm. Together, these improvements made possible the collection of a significant amount of data. The first simply takes advantage of symmetries in the matrix $B$. In many cases that we consider, there is a finite linear group $G$ of automorphisms of the arrangement. Indeed, this circumstance motivated Varchenko to ask the original questions that we investigated. The group $G$ acts on the set of regions and this action commutes with $B$. Let $\chi$ be an irreducible character of $G$ which has rational character values, and let $V_{\chi}$ denote the $\chi$-isotypic component of the action of $G$ on the vector space $V$ spanned by regions. Since the actions of $B$ and $G$ commute, $B$ preserves the subspace $V_{\chi}$. Let $B_{\chi}$ denote the restriction of $B$ to $V_{\chi}$. The similarity which transforms $B$ into the direct sum of the $B_{\chi}$ is defined over $\mathbf{Q}$ hence can be taken to be a unimodular matrix over $\mathbf{Q}[q]$. So, the Smith Normal Forms of $B$ and $\oplus B_{\chi}$ (over $\mathbf{Q}[q]$ ) are equal. There is additional structure to the matrix $B_{\chi}$. It is known well-known that $B_{\chi}$ can be written as a direct sum of $d_{\chi}$ copies of a square matrix $P_{\chi}$, of size $m_{\chi}$ where $d_{\chi}$ is the degree the representation $\chi$ and $m_{\chi}$ is the multiplicity of $\chi$ in the representation $V$. Moreover, the matrix $P_{\chi}$ can be computed as the restriction of $B$ to the image of a primitive idempotent $E_{\chi}$ corresponding to the character $\chi$ in the group algebra $\mathbf{C} G$. So, if an idempotent $E_{\chi}$ can be found which is defined over $\mathbf{Q}$, then the collection of polynomials that occur on the diagonal of the $\operatorname{SNF}(B)$ is equal to the collection of polynomials that occur on the diagonal of the direct sum of the Smith Normal Forms of the $P_{\chi}$ (each $P_{\chi}$ repeated $d_{\chi}$ times).

For example, consider the case where $\mathcal{A}$ is the braid arrangement $\mathbf{A}_{n-1}$. In this case, the matrix $B$ is 720 by 720 and so direct computation of its Smith Normal Form is intractable. The group $G=S_{6}$ acts on the arrangement $\mathcal{A}$ and it is well-known that there exist primitive idempotents corresponding to the irreducible representations of $G$ which are defined over $\mathbf{Q}$. The irre- 
ducible representations of $G$ are indexed by partitions of 6 . In this case, the representation of $G$ on $V$ is the regular representation so the multiplicity $m_{\chi}$ is equal to the degree $d_{\chi}$. Amongst partitions of 6 , the maximum value of $m_{\chi}=d_{\chi}$ is 16 for the partition $\chi=321$. So for $n=6$, the computation of the Smith Normal Form of the original matrix of size 720 has been reduced to the problem of computing the Smith Normal Forms of eleven matrices of sizes 16 and less. This represents an enormous improvement in computational complexity.

Even with this improvement, the computation of the Smith Normal Form of the matrix $B$ remains intractable in many cases because of the degree blow-up phenomenon discussed above. For example, the computation of the Smith Normal Form of the matrix $P_{42}$ (as defined in the previous paragraph) suffers from the blow-up of degrees at intermediate points in the computation. This was in fact the 9 by 9 matrix with entries of degree 15 or less that was discussed above. We need a second idea to get around this degree blow-up problem.

Suppose we are trying to compute the Smith Normal Form over $\mathbf{Q}[q]$ of the matrix $M$. Assume in addition that we know the determinant of $M$ (in our case we will know the determinant of $B$ because of Varchenko's Theorem). Let $\psi(q)$ be an irreducible polynomial over $\mathbf{Q}$ of degree $\delta$ and let $N$ denote the multiplicity of $\psi(q)$ as a factor of $\operatorname{det}(M)$. We can expand any polynomial $p(q)$ as a sum of powers of $\psi(q)$ with coefficients that are polynomials of degree less than $\delta$. The idea of our second improvement is to throw away the tail in this $\psi(q)$-expansion throughout the computation for each entry of $M$, i.e., for each entry $M_{i, j}$ we will throw away that part of the expansion which involves powers of $\psi(q)$ higher than $F$ for some fixed $F$.

To make this more precise, fix an irreducible $\psi(q)$ of degree $\delta$ and fix $F$. Let $p(q)$ be a polynomial with $\psi(q)$-expansion

$$
p(q)=\sum_{r \geq 0} a_{r}(q) \psi(q)^{r}
$$

where each $a_{r}(q)$ is a polynomial of degree less than $\delta$. Define $\phi_{F}(p(q))$ to be

$$
\phi_{F}(p(q))=\sum_{r=0}^{F-1} a_{r}(q) \psi(q)^{r} .
$$

Extend the notation $\phi_{F}$ to matrices by saying that $\left(\phi_{F}(M)\right)_{i, j}=\phi_{F}\left(M_{i, j}\right)$. For any matrix $M$ we have

$$
M=\phi_{F}(M)+\psi(q)^{F} M^{\prime}
$$


for some other matrix $M^{\prime}$. When you follow the apply the usual algorithm (described above) for computing the Smith Normal Form of a matrix $M$, you obtain a sequence of matrices, $M=M_{0}, M_{1}, \ldots, M_{k}=\operatorname{SNF}(M)$ where each $M_{i+1}$ is obtained from the previous $M_{i}$ by either left or right multiplication by a unimodular matrix $E_{i}$. Consider the new algorithm, which we call the $\phi_{F}$-algorithm which proceeds in exactly the same way, except that after each step you apply $\phi_{F}$ to the outcome of the previous step before proceeding. In essence, all that the $\phi_{F}$-algorithm does is to compute the Smith Normal Form of our original matrix over the ring $\mathbf{Q}[q] /\left(\psi(q)^{F}\right)$. We will denote this matrix by $\mathrm{SNF}_{\psi(q), F}(M)$. Note that $\mathrm{SNF}_{\psi(q), F}(M)$ is diagonal and that each entry divides the previous entry modulo $\psi(q)^{F}$. It is instructive to see an example at this point.

Example 2.2. Let $\psi=\psi(q)=1+q+q^{2}$ and let $M$ be the matrix

$$
M=\left(\begin{array}{cc}
1-q-3 q^{2}-6 q^{3}-4 q^{4}-2 q^{5} & 2 q+4 q^{2}+6 q^{3}+4 q^{4}+2 q^{5} \\
-q-2 q^{2}-3 q^{3}-2 q^{4}-q^{5} & 1+2 q+3 q^{2}+2 q^{3}+q^{4}
\end{array}\right) .
$$

It is straightforward to check that the Smith Normal Form of $M$ over $\mathbf{Q}[q]$ is

$$
\operatorname{SNF}(M)=\left(\begin{array}{cc}
\psi & 0 \\
0 & \psi^{2}
\end{array}\right)
$$

Now let's see what we get if we apply the $\phi_{F}$-algorithm for various values of $F$. If we expand each entry of the original $M$ in terms of powers of $\psi$ we obtain

$$
M=\left(\begin{array}{cc}
\psi+2(1+q) \psi^{2}-2 \psi^{3} & 2 q \psi^{2} \\
-q \psi^{2} & \psi^{2}
\end{array}\right) .
$$

So if we apply the $\phi_{1}$-algorithm to $M$ we see at the very first step

$$
\phi_{1}(M)=\left(\begin{array}{ll}
0 & 0 \\
0 & 0
\end{array}\right)
$$

which is already in Smith Normal Form. So $\operatorname{SNF}_{\psi, 1}(M)$ is the zero matrix.

Similarly, when we apply $\phi_{2}$ to $M$ we obtain a matrix that is already in Smith Normal Form:

$$
\phi_{2}(M)=\operatorname{SNF}_{\psi, 2}(M)=\left(\begin{array}{ll}
\psi & 0 \\
0 & 0
\end{array}\right) .
$$

Finally, when we apply the $\phi_{3}$-algorithm to $M$ we go through the following series of steps to arrive at $\mathrm{SNF}_{\psi, 3}(M)$ :

$$
\left(\begin{array}{cc}
\psi+2(1+q) \psi^{2} & 2 q \psi^{2} \\
-q \psi^{2} & \psi^{2}
\end{array}\right)
$$


Right multiply by a unimodular to get:

$$
\left(\begin{array}{cc}
\psi+2(1+q) \psi^{2} & -4 q(1+q) \psi^{3} \\
-q \psi^{2} & \psi^{2}+2 q^{2} \psi^{3}
\end{array}\right) .
$$

Apply $\phi_{3}$ to get:

$$
\left(\begin{array}{cc}
\psi+2(1+q) \psi^{2} & 0 \\
-q \psi^{2} & \psi^{2}
\end{array}\right)
$$

Left multiply by a unimodular to get:

$$
\left(\begin{array}{cc}
\psi+2(1+q) \psi^{2} & 0 \\
0 & \psi^{2}
\end{array}\right)
$$

This last matrix is $S N F_{\psi, 3}(M)$. Note that successive diagonal entries do not divide each other in $\mathbf{Q}[q]$ but do divide each other modulo $\psi^{3}$. Also note that we had no problem with exponent growth when we computed $\mathrm{SNF}_{\psi, F}(M)$. In fact, the maximum exponent that we can encounter during the $\phi_{F}$-algorithm is something like $2 \delta F$.

Recall that $m_{i}(\psi)$ denotes the power of $\psi$ which exactly divides the $i^{\text {th }}$ diagonal entry of $\operatorname{SNF}(M)$. Let $m_{i, F}(\psi)$ denote the corresponding power of $\psi$ which exactly divides the $i^{\text {th }}$ diagonal entry of $\operatorname{SNF}_{\psi, F}(M)$. We claim that $m_{i, F}(\psi)=m_{i}(\psi)$ if $m_{i}(\psi)$ is less than $F$. Otherwise, $m_{i, F}(\psi)=0$.

To see this, let $V$ be $\mathbf{Q}[q]^{n}$. We can think of $M$ as a map from $V$ to $V$. The significance of the exponents $m_{i}(\psi)$ is that we can choose $v_{1}, \ldots, v_{n}$, with each $v_{i}$ having relatively prime coordinates, such that

$$
M v_{i} \subset\left(\psi^{m_{i}(\psi)}\right) V
$$

Similarly, the significance of the exponents $m_{i, F}(\psi)$ is that we can choose $x_{1}, \ldots, x_{n}$, such that

$$
M x_{i} \subset\left(\psi^{m_{i, F}(\psi)}\right) V+\oplus\left(\psi^{F}\right) V
$$

So if we choose $F$ to be greater than or equal to the maximum of the original $m_{i}(\psi)$, we will have $m_{i}(\psi)=m_{i, F}(\psi)$ for all $i$. In practice, one has to determine which value of $F$ to use since you don't know a priori the maximum value of the $m_{i}(\psi)$. The reader will note that Conjecture 3.3 stated in the next section exactly answers this question. But until that conjecture is proved, there remains some element of trial and error in choosing an appropriate value of $F$. It is easy to tell if you have chosen $F$ large enough because in that case, the sum of the $m_{i, F}(\psi)$ equals the exact power of $\psi$ which divides the determinant of $M$. In our computational work, we used 
Conjecture 3.3 as a guide in choosing $F$ and that led us to the optimal value of $F$ in every case.

How well does this algorithm work? Recall that we previously mentioned an example in which $\mathrm{M}$ was a nine by nine matrix with entries of degree fifteen or less. In that case the usual algorithm for computing the Smith Normal Form of this matrix experienced severe exponent blow-up and had failed to finish after a week of computation. However, using the $\phi_{F}$-algorithm, there was a striking speed-up. For each irreducible $\psi$, the computation of the $m_{i, F}(\psi)$ took less than five seconds. In this case, Varchenko's determinant formula told us that there were ten irreduibles $\psi$ that needed to be considered. So the entire computation of the Smith Normal Form which had failed to run in a week's time using the standard algorithm, took less than a minute using the $\phi_{F}$-algorithm. In the Appendix we present some of the results of our computations.

\section{Conjectures and Results.}

In the last section, we described algorithms that we implemented to compute the Smith Normal Form of the Varchenko $B$ matrices in a number of cases. In the Appendix, the reader will find some of the results of the actual computations. These computational results led to a number of conjectures and suggested a number of questions. In this section we state two conjectures that are based on these computations. We will prove one of the two conjectures in the next section.

We will need one piece of notation to state the first result. Let $\mathcal{A}$ be an arrangement and $B$ the associated Varchenko matrix. For $\psi$ an irreducible polynomial, let $t_{\psi, i}$ denote the number of diagonal entries in $\operatorname{SNF}(B)$ that are exactly divisible by $\psi^{i}$. In the notation of the previous section, $t_{\psi, i}$ is the number of $m_{j}(\psi)$ that are equal to $i$.

Theorem 3.1. Let $\psi=q-1$. Then $t_{\psi, i}$ is equal to the dimension of the $i^{\text {th }}$ graded piece of the Orlik-Solomon algebra of $\mathcal{A}$. Equivalently, $t_{\psi, i}$ is equal to the dimension of the $i^{\text {th }}$ graded piece of the singular homology of $\mathbf{C}^{n}-\mathcal{A}_{\mathbf{C}}$ where $\mathcal{A}_{\mathbf{C}}$ denotes the complexification of the arrangement A. Equivalently,

$$
t_{\psi, i}=\sum_{\rho(X)=i}(-1)^{i} \mu(0, X)
$$

where the sum is over elements $X$ in the intersection lattice of $\mathcal{A}$.

This theorem is the main result of this paper and will be proved in the next section. It immediately suggests a question: Is there a similarly elegant 
description of the numbers $t_{\psi, i}$ for other irreducibles $\psi$ ? At this point, the authors do not know such a description. The reader is invited to speculate using the data that appears in the last section.

The next conjecture is less elegant but is the fact needed by Hanlon and Stanley to complete the proof of their conjectures in [2]. This conjecture also provides the information needed to optimally set a parameter $F$ for the $\phi_{F}$-algorithm discussed in the previous section. It concerns the maximum value of $m_{j}(\psi)$ for a given irreducible $\psi$. Let $\operatorname{Height}(\psi, \mathcal{A})$ denote this maximum value for the arrangement $\mathcal{A}$. We will need a definition to state the conjecture.

Definition 3.2. Let $X$ be an edge in the intersection lattice of $\mathcal{A}$ and let $\psi$ be an irreducible. Recall from Varchenko's determinant formula that $X$ contributes a factor of the form $\left(1-q^{2 h(X)}\right)^{\ell(X)}$ where $h(X)$ is the number of hyperplanes that contain $X$ and $\ell(X)$ is an exponent described in Section 1. We say that $X$ is $\psi$-active if

1. $\psi$ divides $1-q^{2 h(X)}$.

2. The exponent $\ell(X)$ is nonzero.

If $L(\mathcal{A})$ contains no $\psi$-active elements, then $\operatorname{Height}(\psi, \mathcal{A})$ is zero. Otherwise, let

$$
\operatorname{Max}(\psi, \mathcal{A})=\max _{U} \sum_{X \in U} \operatorname{Height}\left(\psi, \mathcal{A}_{X}\right)
$$

where the maximum is taken over all sets $U \subseteq L(\mathcal{A}) \backslash\{1\}$ having the property, for distinct $X, Y \in U$, that $X \wedge Y=0$.

Conjecture 3.3. Let $\psi$ be an irreducible polynomial. Then $\operatorname{Height}(\psi, \mathcal{A})$ is given recursively as follows:

$$
\operatorname{Height}(\psi, \mathcal{A})= \begin{cases}1+\operatorname{Max}(\psi, \mathcal{A}) & \text { if } 1 \in L(\mathcal{A}) \text { is } \psi \text {-active } \\ \operatorname{Max}(\psi, \mathcal{A}) & \text { otherwise }\end{cases}
$$

An interesting special case to consider is the case where the $\psi$-active edges in $L$ form an antichain, and no two such edges span the whole space $\mathbf{R}^{n}$. In this case, Conjecture 3.3 asserts that the maximum value of $m_{j}(\psi)$ should be 1. Equivalently, when $q$ is chosen to be a root of $\psi, B(q)$ show have a nullspace of dimension $\sum_{X} \ell(X)$, where the sum is taken over all edges $X$ in this antichain.

\section{The Main Result.}

In this section we will prove Theorem 3.1 stated above. Our proof will involve some background results which we establish first. 
We shall need a notion of orientation that applies to all affine subspaces $X$ of $\mathbf{R}^{n}$. Let $\left(\tau_{1}, \tau_{2}, \ldots, \tau_{n}\right)$ be a fixed, ordered basis of $\mathbf{R}^{n}$ in general position with respect to $\mathcal{A}$. That is, for any affine subspace $X \in L_{k}(\mathcal{A})$ and any subset of the basis $\left\{\tau_{i_{1}}, \ldots, \tau_{i_{r}}\right\}$, we have

$$
\operatorname{dim} \operatorname{span}\left\{X \cup\left\{\tau_{i_{1}}, \ldots, \tau_{i_{r}}\right\}\right\}=\min \{n, r+\operatorname{dim} X\},
$$

where vectors in $X$ are considered as vectors of the corresponding linear subspace. Then, for any ordered set of independent vectors $\left(x_{1}, x_{2}, \ldots, x_{n-k}\right)$ in an affine space $X \in L_{k}$, we define their orientation to be the sign of $\operatorname{det}\left(\tau_{1}, \ldots, \tau_{k}, x_{1}, \ldots, x_{n-k}\right)$ : For convenience, let

$$
\tau\left(x_{1}, \ldots, x_{n-k}\right)=\frac{\operatorname{det}\left(\tau_{1}, \ldots, \tau_{k}, x_{1}, \ldots, x_{n-k}\right)}{\left|\operatorname{det}\left(\tau_{1}, \ldots, \tau_{k}, x_{1}, \ldots, x_{n-k}\right)\right|} .
$$

From here through to Proposition 4.4 we shall summarize some results from Varchenko and Gel'fand [7]. Let $M$ be the complex vector space spanned by $r(\mathcal{A})$. (By way of comparison, $V$ was the $\mathbf{C}[q]$-module spanned by $r(\mathcal{A})$, so one can view $M$ as being included in $V$.) Let $T=M^{*}$, its dual space. Now, for each hyperplane $H \in \mathcal{A}$, fix a functional $\alpha_{H} \in\left(\mathbf{R}^{n}\right)^{*}$ whose set of zeroes is exactly $H$. The Heaviside function $x_{H} \in T$ is defined as follows. On a basis vector $R \in r(\mathcal{A})$,

$$
x_{H}(R)= \begin{cases}0 & \text { if } \alpha_{H}(v)<0 \text { for some (any) } v \in R, \\ 1 & \text { if } \alpha_{H}(v)>0 .\end{cases}
$$

We remark that $x_{H}$ is idempotent for each $H \in \mathcal{A}$, and that products of Heaviside functions can be regarded as characteristic functions on the regions inside a cone that is bounded by a set of hyperplanes. Varchenko and Gel'fand [7] prove the following.

Proposition 4.1. Suppose a set of hyperplanes $H_{1}, \ldots, H_{p}$ is linearly dependent. Let

$$
\sum_{i} c_{i} \alpha_{H_{i}}=0
$$

for some nonzero constants $\left\{c_{i}\right\}$. Let $J_{+}=\left\{H_{i}: c_{i}>0\right\}$, and $J_{-}=$ $\left\{H_{i}: c_{i}<0\right\}$. Then in the ring $T$,

$$
\prod_{H \in J_{+}} x_{H} \prod_{H \in J_{-}}\left(x_{H}-1\right)-\prod_{H \in J_{-}} x_{H} \prod_{H \in J_{+}}\left(x_{H}-1\right)=0 .
$$

Conversely, they show that these relations, together with the idempotence of the generators, characterizes $T$ :

Theorem 4.2. The ring map $\pi: \mathbf{C}\left[u_{H}: H \in \mathcal{A}\right] \rightarrow T$ induced by $\pi\left(u_{H}\right)=x_{H}$ is surjective. The kernel of $\pi$ is an ideal generated by

$$
\left\{x_{H}^{2}-x_{H}: H \in \mathcal{A}\right\}
$$


and by relations of the form $(*)$ induced by all sets $\left\{H_{1}, \ldots, H_{p}\right\} \subseteq \mathcal{A}$ of linearly dependent hyperplanes.

It makes sense, then, to define the degree of any $f \in T$ as the smallest degree of a polynomial in the generators above that equals $f$. Filter $T$ by degree by setting $T^{k}=\{f \in T: \operatorname{deg} f \leq k\}$. Since the generators are idempotent, it must be the case that $T=T^{n}$. Thus we have

$$
T=T^{n} \supseteq T^{n-1} \supseteq \cdots \supseteq T^{0} \supset 0 .
$$

One can define a graded map $\phi: \operatorname{Gr}(T) \rightarrow A(\mathcal{A})$ as follows. On monomials, $\phi^{p}: T^{p} / T^{p-1} \rightarrow A^{p}(\mathcal{A})$ via

$$
\phi^{p}\left(x_{H_{1}} \cdots x_{H_{l}}\right)= \begin{cases}0 & \text { if } l<p \\ \tau\left(\alpha_{H_{1}}, \ldots, \alpha_{H_{l}}\right) a_{H_{1}} \wedge \cdots \wedge a_{H_{l}} & \text { if } l=p .\end{cases}
$$

To verify that $\phi$ is well-defined, one checks that ker $\pi$ from Theorem (4.2) is mapped to zero in the the Orlik-Solomon algebra $A(\mathcal{A})$. Since $\phi$ is surjective and $\operatorname{dim} A(\mathcal{A})=|r(\mathcal{A})|=\operatorname{dim} T, \phi$ is in fact a vector space isomorphism. We see that $A_{k} \cong T^{k} / T^{k-1}$, which means that

$$
\operatorname{dim} T^{k}-\operatorname{dim} T^{k-1}=\left[t^{k}\right] \pi(\mathcal{A}, t),
$$

the coefficient of $t^{k}$ in the polynomial $\pi(\mathcal{A}, t)$.

Recall that $M$ was the vector space based on the set of regions. The filtration of $T$ induces a filtration of $M$ given by defining $M_{k}=\left(T^{k-1}\right)^{\perp}$ for $0<k \leq n$, and $M_{0}=M$ :

$$
M=M_{0} \supseteq M_{1} \supseteq \cdots \supseteq M_{n} \supset 0 .
$$

Our conclusion is the following:

Proposition 4.3. $\operatorname{dim} M_{k}-\operatorname{dim} M_{k+1}=\left[t^{k}\right] \pi(\mathcal{A}, t)$.

4.1. Some explicit elements of $M$. We must introduce the flag complex from [7]. Let $\mathcal{F}_{k}$ be the complex vector space with the following basis:

$$
\begin{aligned}
& \left\{\left(X_{0}, X_{1}, \ldots, X_{k}\right): X_{0}=\mathbf{R}^{n}, X \in L(\mathcal{A}),\right. \\
& \left.\quad \text { and } X_{i-1} \supseteq X_{i}, \rho X_{i}=i \quad \text { for } 1 \leq i \leq k\right\} .
\end{aligned}
$$

Call $\mathcal{F}_{k}$ the space of flags of dimension $k$. 
Now define some elements of $M$ that are indexed by flags and regions. Let $F=\left(X_{0}, \ldots, X_{k}\right)$ be a flag of dimension $k$, and $Q \in r\left(\mathcal{A}^{X_{k}}\right)$ a region. Define the $\operatorname{brick}^{1} \varphi(F, Q) \in M$ :

$$
\varphi(F, Q)=\sum_{U \subseteq[k]}(-1)^{|U|} Q_{U},
$$

where $Q_{U}$ is an element of $r(\mathcal{A})$ determined from $Q, F$, and $U$ as follows. Let $\beta_{k}$ be a vector in $X_{k-1} \backslash X_{k}$. Choose the sign of $\beta_{k}$ so that the orientation $\tau\left(\beta_{k}\right)$ is positive. Proceed inductively to choose a vector $\beta_{i}$ for each $1 \leq i \leq k$ for which $\beta_{i}$ lies in $X_{i-1}$ and not $X_{i}$, and for which $\tau\left(\beta_{k}, \beta_{k-1}, \ldots, \beta_{i}\right)>0$. Choose an arbitrary point $p \in Q$, and let $Q_{U}(\epsilon)$ be the region containing

$$
p \pm \epsilon \beta_{k} \pm \epsilon^{2} \beta_{k-1} \pm \cdots \pm \epsilon^{k-1} \beta_{1},
$$

for $\epsilon>0$. The sign of $\epsilon^{k-i} \beta_{i}$ is taken to be negative if $i \in U$, and positive otherwise. As $\epsilon$ approaches zero, one can see that $Q_{U}(\epsilon)$ is eventually constant. Call this region $Q_{U}$. By construction, it is also independent of the choice of $p$.

By way of an example, let $\tau_{1}=(2,1)$ and $\tau_{2}=(-1,2)$. Let $\mathcal{A}=\left\{\ell_{1}, \ell_{2}, \ell_{3}\right\}$, where

$$
\begin{aligned}
\ell_{1} & =\{(x, y): x=0\}, \\
\ell_{2} & =\{(x, y): y=0\}, \quad \text { and } \\
\ell_{3} & =\{(x, y): x=y\} .
\end{aligned}
$$

Let $F=\left(\mathbf{R}^{2}, \ell_{1}, 0\right)$; then by choosing $\beta_{2}=(1,0)$ and $\beta_{1}(0,1)$, we find that $\varphi(F, 0)$ is supported on the following regions:

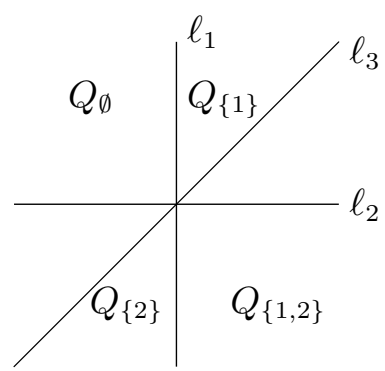

By checking that any monomial in $T^{k-1}$ is zero when applied to $\varphi(F, Q)$, one sees that $\varphi(F, Q)$ is contained in $M_{k}$. Let

$$
\mathcal{B}_{k}=\left\{\varphi(F, Q): F \in \mathcal{F}_{k}, Q \in r\left(\mathcal{A}^{X_{k}}\right)\right\},
$$

and $\mathcal{B}=\cup_{k} \mathcal{B}_{k}$, the set of all bricks. One finds:

Proposition 4.4 ([7]). The set of bricks $\mathcal{B}$ spans $M$. Specifically, $\mathcal{B}_{k}$ spans $M_{k}$.

\footnotetext{
${ }^{1}$ A flag cochain in [7] and [8].
} 
4.2. Applying matrix $B$ to the bricks. This subsection relates $\left(1-q^{2}\right)^{k}$ in $\operatorname{SNF}(B)$ to the spaces $M_{k}$, through the following proposition, which is similar to [6, Lemma 20.1.1].

Proposition 4.5. $(1-q)^{k}$ divides Bx, for each $x \in M_{k}$.

In view of Proposition 4.3, this is almost enough to conclude that the multiplicity of $(1-q)^{k}$ in $\operatorname{SNF}(B)$ is $\left[t^{k}\right] \pi(\mathcal{A}, t)$, as claimed. (The multiplicity of $(1+q)^{k}$ is the same.) By the familiar properties of the Smith Normal Form, one also needs to know that the multiplicity of $1-q$ as a factor of $\operatorname{det} B$ is exactly $\sum_{k} k\left[t^{k}\right] \pi(\mathcal{A}, t)$ (and no greater); this will be shown in Subsection 4.3.

The proof of the proposition will follow some short lemmas. Since the set of bricks spans $M_{k}$ (Proposition 4.4), it is enough to show that $(1-q)^{k}$ divides $B \cdot \varphi(F, Q)$, for each brick $\varphi(F, Q)$ of dimension $k$. Recall $q^{n(R, S)}$ is the $(R, S)$-entry of $B$. For a fixed brick $\varphi(F, Q) \in \mathcal{B}_{k}$, we shall establish some more notation.

Let $C=X_{k}$, the smallest subspace in the flag $F$. Then $\mathcal{A}_{C}$ is the central arrangement containing $C$. Define a function $\phi:[k] \rightarrow 2^{\mathcal{A}_{C}}$ by letting $\phi(i)$ equal the set of hyperplanes in $\mathcal{A}$ that lie between regions $Q_{\emptyset}$ and $Q_{\{i\}}$. Note that $\phi(i)$ is actually contained in $\mathcal{A}_{C}$ for each $i$.

Lemma 4.6. The sets $\{\phi(i): i \in[k]\}$ partition $\mathcal{A}_{C}$.

Proof. For any hyperplane $H \in \mathcal{A}_{C}$, let $j$ be the smallest integer for which $H \supseteq X_{j}$. (Clearly $1 \leq j \leq k$.) From the definition of $Q_{U}$, one can see that the $j^{\text {th }}$ sign is the only one which effects that side of $H$ on which $Q_{U}$ lies. That is, $H \in \phi(j)$, but $H \notin \phi(i)$ for $i \neq j$.

Now extend $\phi$ to a map $\Phi: 2^{[k]} \rightarrow 2^{\mathcal{A}_{C}}$ by setting $\Phi(U)=\bigcup_{i \in U} \phi(i)$. Lemma 4.6 states that this is a disjoint union. We should interpret $\Phi(U)$ as the set of hyperplanes separating $Q_{\emptyset}$ and $Q_{U}$.

Lemma 4.7. Let $R$ and $R^{\prime}$ be regions of $\mathcal{A}_{C}$. Let $\mathcal{H}$ and $\mathcal{H}^{\prime}$ be the sets of hyperplanes separating $Q_{\emptyset}$ from $R$ and $R^{\prime}$, respectively. Then the set of hyperplanes separating $R$ from $R^{\prime}$ equals $\mathcal{H} \triangle \mathcal{H}^{\prime}$.

Proof. Let $H \in \mathcal{A}_{C}$ be a hyperplane. Of the three regions $Q_{\emptyset}, R$, and $R^{\prime}$, either all three lie on the same side of $H$, or $H$ separates one from the other two. Thus, $H$ separates $R$ from $R^{\prime}$ if and only if it lies in $\mathcal{H}$ or $\mathcal{H}^{\prime}$, but not both.

Now we are in a position to prove Proposition 4.5. The lemmas make it possible to calculate each component of $B \cdot \varphi(F, Q)$ explicitly, and we shall 
do so. Say

$$
B \cdot \varphi(F, Q)=\sum_{R \in r(\mathcal{A})} a_{R} R,
$$

for some coefficients $\left\{a_{R}\right\}$. For a given $R \in r(\mathcal{A})$, move to a new region $R_{C} \in$ $r(\mathcal{A})$, adjacent to $Q$, without crossing the hyperplanes of $\mathcal{A}_{C}$. (Formally, $R_{C}$ is chosen to be the unique region for which both $Q \subseteq \overline{R_{C}}$ and $R$ and $R_{C}$ lie in the same region of $\mathcal{A}_{C}$.) We have

$$
\begin{aligned}
a_{R} & =\sum_{S \in r(\mathcal{A})} q^{n(R, S)}[\varphi(F, Q)]_{S}, \\
& =\sum_{U \subseteq[k]} q^{n(R, S)}(-1)^{|U|}, \quad \text { by definition of } \varphi(F, Q) \\
& =q^{n\left(R, R_{C}\right)} \sum_{U \subseteq[k]}(-1)^{|U|} q^{n\left(R_{C}, Q_{U}\right)},
\end{aligned}
$$

where the last step uses the fact that $R$ and $R_{C}$ lie in the same region of $\mathcal{A}_{C}$.

Now let $\mathcal{H} \subseteq \mathcal{A}_{C}$ be the set of hyperplanes separating $Q_{\emptyset}$ and $R_{C}$. The second lemma interprets the number of hyperplanes between $R_{C}$ and $Q_{U}$, so that the equality above becomes

$$
\begin{aligned}
a_{R} & =q^{n\left(R, R_{C}\right)} \sum_{U \subseteq[k]}(-1)^{|U|} q^{|\Phi(U) \triangle \mathcal{H}|} \\
& =q^{n\left(R, R_{C}\right)+|\mathcal{H}|} \sum_{U \subseteq[k]}(-1)^{|U|} q^{|\Phi(U)|-2|\Phi(U) \cap \mathcal{H}|} \\
& \text { since }|A \triangle B|=|A|+|B|-2|A \cap B|, \\
& =q^{n\left(R, R_{C}\right)+|\mathcal{H}|} \prod_{i \in[k]}\left(1-q^{|\phi(i)|-2|\phi(i) \cap \mathcal{H}|}\right)
\end{aligned}
$$

$(1-q)$ divides each term of the product, (which of course may be zero), so $(1-q)^{k}$ divides the coefficient $a_{R}$. This completes the proof.

4.3. $1-q^{2}$ in the Determinant of $B$. As we mentioned before, the argument above only shows that, for each $k,\left[t^{k}\right] \pi(\mathcal{A}, t)$ distinct invariant factors of $B$ are divisible by $\left(1-q^{2}\right)^{k}$. To show further that the multiplicity of $\left(1-q^{2}\right)^{k}$ as an invariant factor of $B$ is exactly $\left[t^{k}\right] \pi(\mathcal{A}, t)$, one can prove the following:

Proposition 4.8. $\quad 1-q^{2}$ divides $\operatorname{det} B$ exactly

$$
\left.\frac{d}{d t} \pi(\mathcal{A}, t)\right|_{t=1}=\sum_{k=0}^{n} k\left[t^{k}\right] \pi(\mathcal{A}, t)
$$

times. 
Following the notation of Orlik and Terao [5], we let $\mathcal{A}_{X}$ denote the hyperplanes of $\mathcal{A}$ that contain a subspace $X$, and and let $\mathcal{A}^{X}$ denote the arrangement contained in a subspace $X$. From Varchenko's determinant formula (1.3), the multiplicity of $1-q^{2}$ in $\operatorname{det} B$ is

$$
\sum_{X \in L(A)} \ell(X)
$$

where $\ell(X)$ is the function introduced in Section 1. It can also be expressed as $\ell(X)=n(X) p(X)$, where $n(X)=\left|r\left(\mathcal{A}^{X}\right)\right|=\pi\left(\mathcal{A}^{X}, 1\right)$, and $p(X)=$ $\chi\left(\mathbf{d} \mathcal{A}_{X}\right)$. Here, $\mathbf{d} \mathcal{A}$ denotes the projectivization or "deconing" of $\mathcal{A}$, and $\chi(\cdot)$ is the Euler characteristic of an arrangement; see [5] for more detail. We remark that Schechtman, Terao, and Varchenko in [9] have identified the number $p(X)$ as Crapo's Beta Invariant of the matroid corresponding to $\mathcal{A}$, which was first defined in [1].

Lemma $4.9([9])$.

For any $X \in L_{k}(\mathcal{A})$, we have $p(X)=\left.(-1)^{k-1} \frac{d}{d t} \pi\left(\mathcal{A}_{X}, t\right)\right|_{t=-1}$.

Proof. By way of comparison, recall that $\chi\left(\mathcal{A}_{X}\right)=(-1)^{k} \pi\left(\mathcal{A}_{X},-1\right)$. The formula is established by viewing the central arrangement $\mathcal{A}_{X}$ as the cone of $\mathbf{d} \mathcal{A}_{X}$ : See [5]. From this perspective, Orlik and Terao show that

$$
\pi\left(\mathcal{A}_{X}, t\right)=(1+t) \pi\left(\mathbf{d} \mathcal{A}_{X}, t\right) .
$$

Since the characteristic polynomial $\chi\left(\mathcal{A}_{X}, t\right)=t^{-k} \pi\left(\mathcal{A}_{X},-t^{-1}\right)$, we find that $\chi\left(\mathbf{d} \mathcal{A}_{X}\right)$ equals

$$
\lim _{t \rightarrow-1}(-1)^{k-1} \pi\left(\mathcal{A}_{X}, t\right) /(1+t),
$$

the derivative at $t=-1$.

Thus we see that $p(X)$ depends only on the semilattice $L(\mathcal{A})$, and only on its elements of rank no greater than $k$. Now we are prepared to prove the proposition.

Proof of Proposition 4.8. The multiplicity of $1-q^{2}$ in $\operatorname{det} B$ is

$$
\sum_{Y} p(Y) n(Y)=\sum_{X \leq Y \leq Z}(-1)^{\rho(Z)} \rho(X) \mu(\hat{0}, X) \mu(Y, Z),
$$

using Lemma 4.9 and the definition of the polynomial $\pi(-, t)$. Here $\mu$ is the Möbius function of the lattice, and $\rho(X)$ is, once again, the rank of $X \in L$. The sum is taken over all ordered triples $X \leq Y \leq Z$ in $L$. Our goal is to show that $(*)$ equals

$$
\sum_{X}(-1)^{\rho(X)} \rho(X) \mu(\hat{0}, X)
$$


Use Möbius inversion as follows. Let $f(X)=\rho(X) \mu(\hat{0}, X)$ for all $X \in L$. Let

$$
S(X, Z)=\sum_{X \leq Y \leq Z} \rho(Y) \mu(X, Y)
$$

and, for short, $S(X)=S(\hat{0}, X)$. Then

$$
S(Y)=\sum_{X \leq Y} f(X)
$$

inverting this gives

$$
f(Y)=\sum_{X \leq Y} \mu(X, Y) S(X)
$$

Thus

$$
\begin{aligned}
(*) & =\sum_{X \leq Y \leq Z}(-1)^{\rho(Z)} f(X) \mu(Y, Z) \\
& =\sum_{Y \leq Z}(-1)^{\rho(Z)} S(Y) \\
& =\sum_{Z}(-1)^{\rho(Z)} f(Z) \\
& =\sum_{Z}(-1)^{\rho(Z)} \rho(Z) \mu(\hat{0}, Z),
\end{aligned}
$$

as required.

\section{Computational Results.}

This final section contains some of the computational results we obtained when we computed the Smith Normal Forms of the Varchenko matrices for various arrangements. This data is presented in the following format. First we write down an arrangement $\mathcal{A}$. The data that follows will give the Smith Normal Form of the Varchenko matrix $B=B(q)$ for the arrangement $\mathcal{A}$. Next, the reader will see the number of regions in the complement of this arrangement and the determinant of the Varchenko matrix $B$. Note that Varchenko's theorem implies that this determinant will be a product of factors of the form $1-q^{S}$ and so any irreducible polynomial dividing the determinant must be a cyclotomic polynomial. Lastly, a table with rows indexed by $d$ and columns indexed by $i$. In this table, the row indices $d$ and column indices $i$ will appear in boldface along the left-hand side and the top. The $d, i$ entry of this table will give the number of diagonal entries in the Smith Normal Form of $B$ which are exactly divisible by $\psi_{d}(q)^{i}$ where $\psi_{d}(q)$ denotes the $d^{t h}$ cyclotomic polynomial. In the notation of the previous 
section, the $d, i$ entry of this table is the number of $m_{j}\left(\psi_{d}(q)\right)$ that are equal to $i$.

Recall from Section 4, that for every $j$ and for every irreducible polynomial $\psi(q), m_{j}(\psi(q))=m_{j}(\psi(-q))$. So for $d$ an odd number, the $d^{t h}$ and $2 d^{t h}$ rows of our table will be identical. Hence we will NOT include the $2 d^{\text {th }}$ rows in the case the $d$ is odd.

To start, we will do a simple example.

Example 5.1. The 3-Fan (also equal to the braid arrangement $B_{3}$ ): Let $A$ consist of the three lines

$$
\begin{aligned}
& y=0 \\
& y=\sin (2 \pi / 3) x \\
& y=\sin (4 \pi / 3) x .
\end{aligned}
$$

There are 6 regions and

$$
\operatorname{det}(B)=\left(1-q^{2}\right)^{6}\left(1-q^{6}\right) .
$$

The Smith Normal Form of $B$ has diagonal entries

$$
\begin{aligned}
& 1 \\
& \left(1-q^{2}\right) \\
& \left(1-q^{2}\right) \\
& \left(1-q^{2}\right) \\
& \left(1-q^{2}\right)^{2} \\
& \left(1-q^{2}\right)\left(1-q^{6}\right) .
\end{aligned}
$$

In the Smith Normal Form there is one entry exactly divisible by $(1-q)^{0}$, three entries exactly divisible by $(1-q)$ and two entries exactly divisible by $(1-q)^{2}$. We indicate that in our chart with row

\section{$\begin{array}{llll}1 & 1 & 3 & 2 .\end{array}$}

We do not include a row in our chart for $d=2$. Next, let $\psi(q)=q^{2}+q+1$ which is the third cyclotomic polynomial. There are five entries in $\operatorname{SNF}(B)$ that are exactly divisible by $\psi(q)^{0}$ and one entry exactly divisible by $\psi(q)$. We denote that in our chart by the row

$$
\begin{array}{llll}
3 & 5 & 1 & 0 .
\end{array}
$$

We do not include a row in our chart for $d=6$. So in this case our chart looks like: 


\section{d/i 012 \\ $\begin{array}{llll}1 & 1 & 3 & 2\end{array}$ \\ $\begin{array}{lllll}3 & 5 & 1 & 0 .\end{array}$}

In what follows, we will present data for two infinite families of arrangements.

The n-Fan Arrangement $F_{n}$ : This is the arrangement in $\mathbf{R}^{2}$ consisting of the $n$ lines $\left\{\ell_{0}, \ldots, \ell_{n-1}\right\}$ where $\ell_{j}$ is given by the equation $y=\sin (2 \pi j / n) x$. In this case the determinant of $B$ is given by

$$
\operatorname{det}(B)=\left(1-q^{2}\right)^{2 n}\left(1-q^{2 n}\right)^{n-2} .
$$

There are $2 n$ regions in the complement of this arrangement.

$\mathrm{n}=1$

d/i 01

$1 \quad 11$

$\mathrm{n}=\mathbf{2}$

d/i 012

1 121

$\mathbf{n}=\mathbf{3}$

d/i 012

1132

$\begin{array}{llll}3 & 5 & 1 & 0\end{array}$

$\mathrm{n}=4$

d/i 012

1143

4620

8620

$\mathrm{n}=\mathbf{5}$

d/i 012

1154

5730. 
Based on our computations in this case we were able to formulate and prove the following result.

Theorem 5.2. The $2 n$ diagonal entries of the Smith Normal Form of $B$ are:

$$
\begin{array}{ll}
1 & (\text { one time }), \\
1-q^{2} & (\text { n times }), \\
\left(1-q^{2}\right)^{2} & (\text { one time }), \\
\left(1-q^{2}\right)\left(1-q^{2 n}\right) & (n-2 \text { times })
\end{array}
$$

Proof. For $\psi=\left(1-q^{2}\right)$ the values of the $m_{i}(\psi)$ given in this theorem follow from Theorem 3.1. For other $\psi$ dividing $1-q^{2 n}$, the result has been proved by one of the authors (G. Denham) as part of more general work which will appear elsewhere.

The braid arrangement $\mathbf{A}_{n-1}$ : Recall from Section 1 that $\mathbf{A}_{n-1}$ is the arrangement in $\mathbf{R}^{n}$ consisting of the $\left(\begin{array}{l}n \\ 2\end{array}\right)$ hyperplanes $H_{i, j}=\left(x_{1}, \ldots, x_{n}\right)$ : $x_{i}=x_{j}$. In this case the determinant of $B$ is given by

$$
\operatorname{det}(B)=\prod_{\ell=2}^{n}\left(1-q^{\ell(\ell-1)}\right)^{\left(\begin{array}{l}
n \\
\ell
\end{array}\right)(\ell-2) !(n-\ell+1) !} .
$$

The Smith Normal Forms of the braid arrangements for various values of $n$ are given by

$$
\mathbf{n}=\mathbf{2}
$$

d/i 01

$\begin{array}{lll}1 & 1 & 1\end{array}$

$\mathbf{n}=\mathbf{3}$

d/i 012

1132

3510

$\mathrm{n}=4$ 
$\mathrm{n}=5$

$$
\begin{array}{cccccc}
\mathbf{d} / \mathbf{i} & \mathbf{0} & \mathbf{1} & \mathbf{2} & \mathbf{3} & \mathbf{4} \\
\mathbf{1} & 1 & 10 & 35 & 50 & 24 \\
\mathbf{3} & 70 & 20 & 30 & 0 & 0 \\
\mathbf{4} & 102 & 10 & 8 & 0 & 0 \\
\mathbf{5} & 114 & 6 & 0 & 0 & 0 \\
\mathbf{1 2} & 100 & 20 & 0 & 0 & 0 \\
\mathbf{2 0} & 114 & 6 & 0 & 0 & 0
\end{array}
$$

$\mathrm{n}=6$

$\begin{array}{ccccccc}\mathbf{d} / \mathbf{i} & \mathbf{0} & \mathbf{1} & \mathbf{2} & \mathbf{3} & \mathbf{4} & \mathbf{5} \\ \mathbf{1} & 1 & 15 & 85 & 225 & 274 & 120 \\ \mathbf{3} & 343 & 106 & 235 & 36 & 0 & 0 \\ \mathbf{4} & 564 & 60 & 96 & 0 & 0 & 0 \\ \mathbf{5} & 654 & 36 & 30 & 0 & 0 & 0 \\ \mathbf{1 2} & 540 & 180 & 0 & 0 & 0 & 0 \\ \mathbf{1 5} & 696 & 24 & 0 & 0 & 0 & 0 \\ \mathbf{2 0} & 648 & 72 & 0 & 0 & 0 & 0 .\end{array}$

As discussed in Section 2, there is an action of the symmetric group which commutes with matrix $B$. So it is possible to write the matrix $B$ as a direct sum:

$$
B=\bigoplus_{\lambda} f_{\lambda} B_{\lambda}
$$

where the sum is over partitions $\lambda$ of $n$ (which index the irreducible representations of $S_{n}$ ), where $f_{\lambda}$ is the dimension of the $S_{n}$-irreducible indexed by $\lambda$ and where $B_{\lambda}$ is the restriction of $B$ to the image of a Young symmetrizer of type $\lambda$. One can then compute the Smith Normal Forms of the individual matrices $B_{\lambda}$. These Smith Normal Forms do not directly give you the Smith Normal Form of $B$, but together they carry the same information, i.e., the dimension of the subspace on which $B$ acts like $\psi(q)^{j}$ for every irreducible $\psi(q)$ and for every $j$. In fact, the collection of $\operatorname{SNF}\left(B_{\lambda}\right)$ tell you more - they give you the $S_{n}$-module structure of the space on which $B$ acts like $\psi(q)^{j}$. Below we give the matrices $\operatorname{SNF}\left(B_{\lambda}\right)$ for some small values of $n$.

$$
\begin{gathered}
\mathbf{n = 2} \operatorname{SNF}\left(B_{2}\right)=(1+q) \\
\operatorname{SNF}\left(B_{1^{2}}\right)=(1-q) \\
\operatorname{SNF}\left(B_{3}\right)=\left((1+q)\left(1+q+q^{2}\right)\right)
\end{gathered}
$$




$$
\left.\begin{array}{c}
\operatorname{SNF}\left(B_{21}\right)=\left(\begin{array}{cc}
1-q^{2} & 0 \\
0 & \left(1-q^{2}\right)^{2}
\end{array}\right) \\
\operatorname{SNF}\left(B_{1^{3}}\right)=\left((1-q)\left(1-q+q^{2}\right)\right) \\
\operatorname{SNF}\left(B_{4}\right)=\left((1+q)\left(1+q+q^{2}\right)\left(1+q+q^{2}+q^{3}\right)\right)
\end{array}\right)
$$

We've also computed this data for $n=5,6$ but omit that data because of its considerable length. With this data, one can compute for each irreducible $\psi$ and each $\mathrm{j}$, the $S_{n}$ module structure of the space where $B$ acts like $\psi^{j}$. Although this character does not seem to be well-behaved for arbitrary $\psi$ and $j$, we believe that it does have a simple description for each $\psi$ when $j$ is taken to be the largest possible value of $j$ so that the corresponding space is

non-zero. However, we have not been able to formulate a precise conjecture which describes this character.

\section{References}

[1] H. Crapo, A higher invariant for matroids, J. Comb. Th., 2 (1967), 406-417.

[2] P. Hanlon and R. Stanley, A q-deformation of a trivial symmetric group action, to appear. 
[3] G. Havas, Recognizing badly presented Z-modules, Lin. Alg. Appl., 192 (1993), 137-163.

[4] M. Newman, Integral Matrices, Academic Press, 1972.

[5] P. Orlik and H. Terao, Arrangements of Hyperplanes, Number 300 in Grundlehren der Mathematischen Wissenschaften, Springer Verlag, 1992.

[6] A.N. Varchenko, Bilinear form of real configuration of hyperplanes, Adv. Math., 97(1) (1993), 110-144.

[7] A.N. Varchenko and I.M. Gel'fand, Heaviside functions of a configuration of hyperplanes, Functional Anal. Appl., 21 (1988), 255-270.

[8] A.N. Varchenko and V.V. Schechtman, Arrangements of hyperplanes and Lie algebra homology, Invent. Math., 106 (1991), 139-194.

[9] A.N. Varchenko, H. Terao and V.V. Schechtman, Local systems over complements of hyperplanes and the Kac-Kazhdan conditions for singular vectors, J. Pure Appl. Algebra, 100(1-3) (1995), 93-102.

[10] D. Zagier, Realizability of a model in infinite statistics, Comm. in Math. Phys., 147 (1992), 199-210.

The second author was partially supported by National Science Foundation Grant No. DMS-9500979.

UNIVERSITY OF MiCHIGAN

ANn Arbor, MI 48109-1109

E-mail address: denham@math.1sa.umich.edu

UNIVERSITY OF MICHIGAN

ANN ARBor, MI 48109-1109

E-mail address: hanlon@math.1sa.umich.edu 